\title{
Preferred Orientation in Stark Rubber
}

\author{
C. J. Newton, L. Mandelkern, and D. E. Roberts
}

\begin{abstract}
The method of pole-figure analysis of preferred orientation is outlined and is applied to the problem of X-ray diffraction from stark rubber. A single nonrotating texture is deduced. The texture indicates that the chain-molecule axis is tilted about $22^{\circ}$ from the plane of the rubber sheet and that a (101) plane normal lies approximately parallel to the sheet.
\end{abstract}

\section{Introduction}

Natural rubber is occasionally found to be hard and inelastic when received or stored in temperate climates. This type of rubber has been designated as "stark rubber", and it is known that its unusual physical properties are due to the development of appreciable amounts of crystallinity. When stark rubber is heated, the observed melting temperature is appreciably higher than that usually assigned to natural rubber. It has been shown recently [1] ${ }^{1}$ that these higher melting temperatures are a consequence of the fact that the crystallites are not randomly arranged relative to one another in stark rubber. These conclusions were based on the X-ray diffraction patterns for several different samples wherein the intensities around the circumferences of diffraction haloes were found to be nonuniform.

If a thin piece of polycrystalline material composed of a very large number of randomly oriented crystallites is placed in a finely collimated X-ray beam, it will cause some of the rays to be diffracted and to form on a photographic film placed normal to the undeviated beam several concentric rings, or haloes, which vary in intensity one from another, but which are uniformly dense each around its own circumference. If the polycrystalline material is made into the form of a fiber and is strained axially, the crystallites will usually no longer be randomly oriented. Each diffraction halo will no longer be uniform but will show broadening and darkening at certain positions, and in extreme cases will break up into a pattern of discrete spots. This is caused by the tendency of the crystallites to approach a "fiber texture", wherein each would ideally have a common crystallographic direction parallel to the axis of the fiber, with radial orientations still random. The X-ray diffraction pattern in such an ideal case would be identical with that from a single crystal of the same material rotating about the fiber direction. Orientations in drawn metal wires and in stretched organic fibers often approach this ideal closely enough that their diffraction patterns may be interpreted by methods similar to those used with rotating single crystal patterns.

When this type of diffraction pattern is observed, the lengths of the spots or areas are related to the

\footnotetext{
1 Figures in brackets indicate the literature references at the end of this paper.
}

angular deviation between the molecular axis direction in the crystallites and the axis of the fiber. A measure of the distribution of orientations can then be obtained from the variation of the intensity along the ares $[2,3]$.

Although the X-ray diffraction haloes from various stark-rubber samples were nonuniform around their circumferences, the patterns did not approach those of fiber-type diagrams. Hence, to describe the preferred orientations of the crystallites in stark rubber, it was necessary to employ a more general method of analysis, the use of the pole figure. Pole-figure analysis, which is explained below, is regularly used in the study of preferred orientation in sheets of metal; but, so far as the authors are aware, it has not heretofore been carried out with a semicrystalline polymer, although a discussion of idealized pole figures for various types of preferred orientation in cellulose has been presented by W. A. Sisson $[4,5]$.

\section{Pole-Figure Analysis}

\subsection{Stereographic Projection}

The pole figure is the conventional method of portraying the angular clustering of certain poles or crystallographic directions with respect to the geometry of the specimen. The means of portrayal is the stereographic projection, which is a two-dimensional representation of the angular relationships between elements of direction in three dimensions. It is based upon the spherical projection, as outlined below.

Let the object to be described, such as the unit cell of a crystal, be placed at the center of a sphere. Lines now drawn from the center, perpendicular to the faces of the cell, will intersect the surface of the sphere when extended. These intersections, or "poles", on the sphere constitute the spherical projection of the unit cell. Angular measurements and symmetry relationships observed among the poles on the sphere reflect the same relationships to be found among the faces of the cell, or the elements of any other entity so projected.

The mapping of the pattern on the sphere onto a plane may be done in several ways. The method we are interested in is the stereographic projection. To visualize this projection, first pass a plane tangent to the reference sphere; this will be known as the plane of projection. Draw a diameter from the point of 
tangency to the opposite side of the sphere. This locates the point of projection, a point on the surface of the sphere diameterically opposite the point of tangency of the plane of projection. Lines are now drawn from the point of projection through the poles on the surface of the reference sphere and extended until they intersect the plane of projection. These intersections result in a pattern on the plane that makes up the stereographic projection. This construction is illustrated in figure 1 .

Poles on that half of the reference sphere opposite the point of projection will project inside the basic circle formed by the projection of the equator of the sphere. Poles on the other hemisphere would project outside the basic circle, but by convention they are usually placed on a second pattern formed by interchanging the point of tangency of the plane and the point of projection, with the points then falling inside the basic circle. If desired, the projections of the two hemispheres may then be superimposed, with some mode of distinction between points if necessary.

The measurement of angles between poles on a stereographic projection is accomplished with the aid of a projection of lines of latitude and longitude from the reference sphere. The chart usually employed for this purpose is known as a Wulff net. Standard projections of crystal plane normals, crystallographic zones, or zone axes are also often used in orientation problems. The general procedure, in brief, is to find suitable rotations about the central point and the meridian on the net that will bring the stereographic projection under examination into coincidence with the standard projection. Details of theory and application of the stereographic projection may be found in Structure of Metals by C. S. Barrett [6].

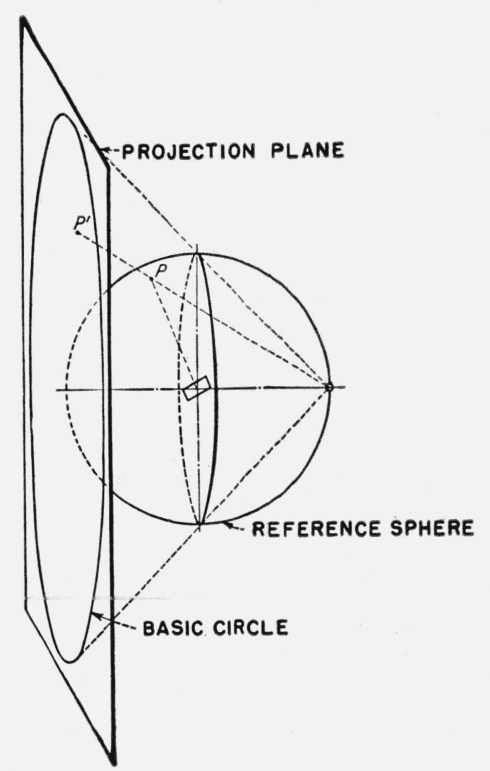

Figure 1. Construction of stereographic projection.

(After C. S. Barrett)

\subsection{Pole Figure}

A pole figure is a graphical representation on a stereographic projection of the clustering of poles relative to the geometry of the specimen. It is developed from the variation in intensity of a certain order of X-ray diffraction at various angles in space around a polycrystalline specimen. The intensity at any point on a diffraction balo is closely related to the number of crystallites oriented in such a manner in the volume of the specimen illuminated by X-rays that they contribute to the diffraction in the direction specified. The pole (i. e., normal) of a crystallographic plane diffracting an X-ray beam bisects the angle between the incident beam and the diffracted beam and lies in the plane they determine. Hence, information read from the diffraction pattern in terms of intensity (corrected if necessary for absorption) may be plotted on a pole figure in such a manner as to represent the increase or decrease over randomness of crystallite orientation in that particular direction.

By reading the intensity at reasonably close increments around a diffraction halo and by systematically varying the angle of incidence of the direct beam upon the specimen, one may plot the distribution of poles for all directions on a pole figure for one family of crystal lattice planes. Then, by combining the pole figures of several orders of diffraction, it may be possible to conclude that there is a single or multiple fiber texture or some other type of preferred orientation system.

\section{Application to Stark Rubber}

\subsection{Experimental Procedure}

The specimen was approximately a 1-mm cube, cut with one edge perpendicular to the surface of a sheet of Hevea rubber designated as sample III in the previous investigation [1]. Reference should be made to this earlier paper for details concerning the preparation and history of the sample. The sheet was microscopically heterogeneous and porous, and the surfaces were extremely rough. The specimen was fixed with water-soluble glue to the end of a thin glass capillary and placed in a collimated beam of $\mathrm{X}$-rays. The $\mathrm{X}$-rays were generated in a copper target tube operated at $30 \mathrm{kv}$ and $26 \mathrm{ma}$. The radiation was filtered by a nickel foil, leaving a predominant radiation maximum of copper $\mathrm{K} \alpha$, with a wavelength of $1.5405 \mathrm{~A}$. An X-ray film was placed 10 $\mathrm{cm}$ beyond the specimen to record the diffraction haloes.

Seven patterns were made at 15 -deg increments of rotation about one cube axis of the specimen. The specimen was then removed, remounted with another cube axis vertical, and the procedure was repeated, after which the third axis was mounted vertical, and again seven exposures were made. In all, 21 patterns were made with stepwise changes in orientation of the specimen with respect to the X-ray beam. In this manner sufficient data for all directions, assuming twofold symmetry, were obtained for the 
pole figures. The patterns were exposed and processed under as nearly constant conditions as practical, so that the densitometer readings, which were then made around each of the three lowest-angle haloes, were comparable from one film to another. A recording microphotometer was used to determine the relative optical densities of the haloes of each film. A special holder was constructed so that the diameter of the film could be scanned; then the film was rotated $10 \mathrm{deg}$ in its plane, and another diameter was scanned. This was repeated until the film had been rotated $180 \mathrm{deg}$. The density distribution for each halo then could be read from the recorder tracings.

The Bragg angle, $\theta$, for each of the three haloes was calculated from the film to specimen distance and the radius of the halo on the film. From this angle and the wavelength, $\lambda$, of the X-rays, one calculated the interplanar spacing $d_{\theta}$, by Bragg's Law:

$$
d_{\theta}=\frac{\lambda / 2}{\sin \theta} .
$$

Indices were assigned to the haloes by matching observed spacings, $d_{\theta}$, with claculated spacings, $d_{h k l}$, arrived at by considering the crystalline rubber to belong to the monoclinic system with lattice parameters $a=12.46 \mathrm{~A}, b=8.89 \mathrm{~A}, c=8.10 \mathrm{~A}$, and $\beta=92 \mathrm{deg}$, as reported by C. W. Bunn [7]. The spacing is calculated from

$$
\frac{1}{d_{h k l}^{2}}=\frac{h^{2}}{a^{2} \sin ^{2} \beta}+\frac{k^{2}}{b^{2}}+\frac{l^{2}}{c^{2} \sin ^{2} \beta}-\frac{2 h l \cos \beta}{a c \sin \beta}
$$

where $h, k$, and $l$ are the order indices of the diffraction produced by the family of atomic planes in question.

The results for the three strongest low-angle haloes were

\begin{tabular}{|c|r|r|r|l|}
\hline Obs. $\theta$ & Sin $\theta$ & $\begin{array}{c}\text { Obs. } d_{\theta} \text { in } \\
\mathrm{A}\end{array}$ & $\begin{array}{c}\text { Calc. } \\
d_{h k l} \text { in } \mathrm{A}\end{array}$ & \multicolumn{1}{c|}{$h k l$} \\
\hline & & & & \\
\hline $7.0^{\circ}$ & 0.1219 & 6.32 & 6.23 & $(200)$ \\
$8.9^{\circ}$ & .1541 & 5.00 & $\begin{array}{l}4.86 \\
5.02\end{array}$ & $\left.\begin{array}{l}(201) \\
(201)\end{array}\right\}$ not resolved \\
$10.5^{\circ}$ & .1822 & 4.23 & 4.19 & $(120)$ \\
& & & & \\
\hline
\end{tabular}

It should be noted that, though the spacing when $h$ and $l$ are of like signs is different from that calculated when $h$ and $l$ are of unlike signs, the diffractions from such slightly different planes may not be resolved. Some uncertainty arises in this matching procedure because of the inherent lack of experimental precision in determining small angles of X-ray diffraction and the presence in a monoclinic structure of numerous spacings whose diffraction maxima are separated by small angular differences. The tables of indices, with calculated and observed intensities, reported by Bunn were of particular value at this point in the problem.

\subsection{Pole-Figure Construction}

As a preliminary to plotting the intensities of a given halo as a pole figure, it is advantageous to make up a set of plotting charts for the diffraction, characterized by its Bragg angle. These plotting guides show, for each setting of the specimen, the traces of the circles on which the poles pertaining to the halo will lie. As the construction of these charts is rather complex, it will not be described here; for details the reader is referred to the excellent article by B. F. Decker [8].

With the aid of the standard charts, the densitometer data for each halo was plotted separately in terms of three magnitudes of intensity. The reliability of the figures so constructed was somewhat reduced because of the lack of very strong variations of intensity around a given halo. It did seem clear, however, that there was a real lack of randomness in the orientations of the diffracting crystallites. Each of the three diffracting haloes showed some changes of intensity, and these changes for each one yielded data that, when plotted in a pole figure for the halo, showed a single region of angular concentration of normals to the plane diffracting.

It was next necessary to construct a standard projection of the monoclinic unit cell of rubber, showing the positions of the normals to the diffracting planes, and certain others, relative to the axes of the crystal. This projection, which may be seen in figure 2, was made by laying out the known configuration of the axes according to Bunn, and by calculating the angular positions of the required plane normals with respect to these axes and plotting them accordingly. In order to see if there existed any mutual relationship of the three pole-figure maxima that would define a single texture in the sheet of rubber, the three individual pole figures were

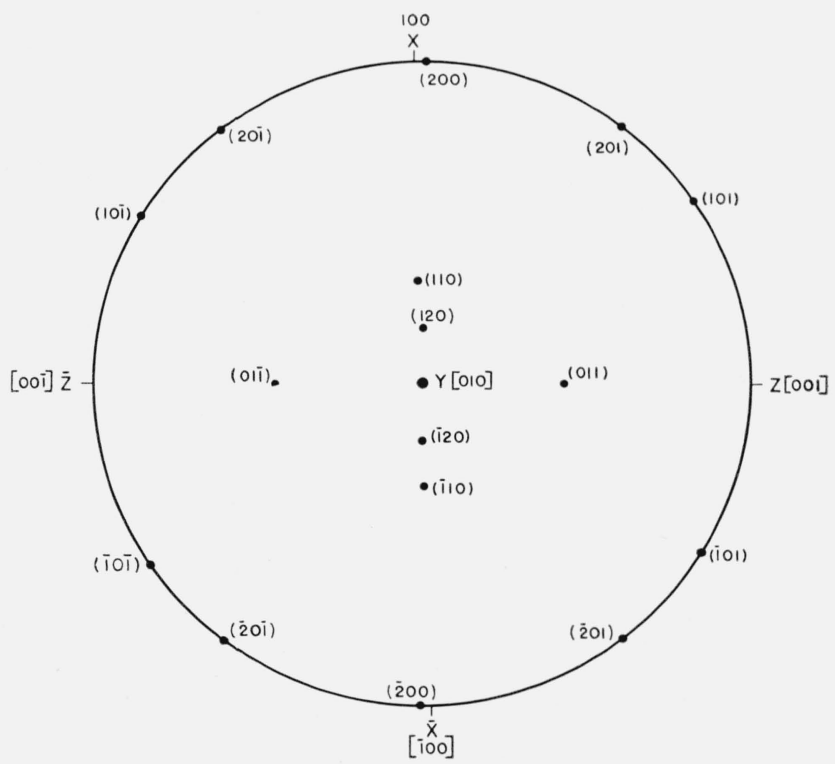

Figure 2. Standard stereographic projection of unit crystalline cell of monoclinic rubber. 
combined on a single stereographic projection for comparison with the standard projection of the unit cell.

\subsection{Interpretation of Pole Figures}

At first examination, the detection or even the existence of a single texture to explain the observed orientations seemed highly unlikely. As stated before, the lack of sharp intensity variations prevented a high degree of positiveness here. Moreover, each of the three haloes on diffraction rings showed a single pole concentration, whereas the standard projection showed multiple poles for the diffracting planes giving rise to the middle and outer rings. Upon close inspection, however, it was found that the spread of each of the regions of secondary intensity on the projection was wide enough to take in its pair of poles, and the single maximum in each case could be explained as the region of their greatest overlap.

If this interpretation of the data is permissible, it does appear that all of the maxima may arise from a single nonrotational texture, characterized by a preference of the $z$-axis (to which the chain molecule is parallel) of the crystallites to cluster about a texture axis, making an angle of about $22^{\circ}$ with the plane of the sheet. This is shown on the stereographic projection in figure 3 . Two qualifications of this specification are to be understood, however: one, the degree of preferred orientation is not very great, only a fraction of the crystallites tending to line up; and, two, the orientation specified for the unit cell is no more than the weighted average orientation, which is subject to the lack of precision, amounting to about $\pm 15^{\circ}$ in any direction on the projection,

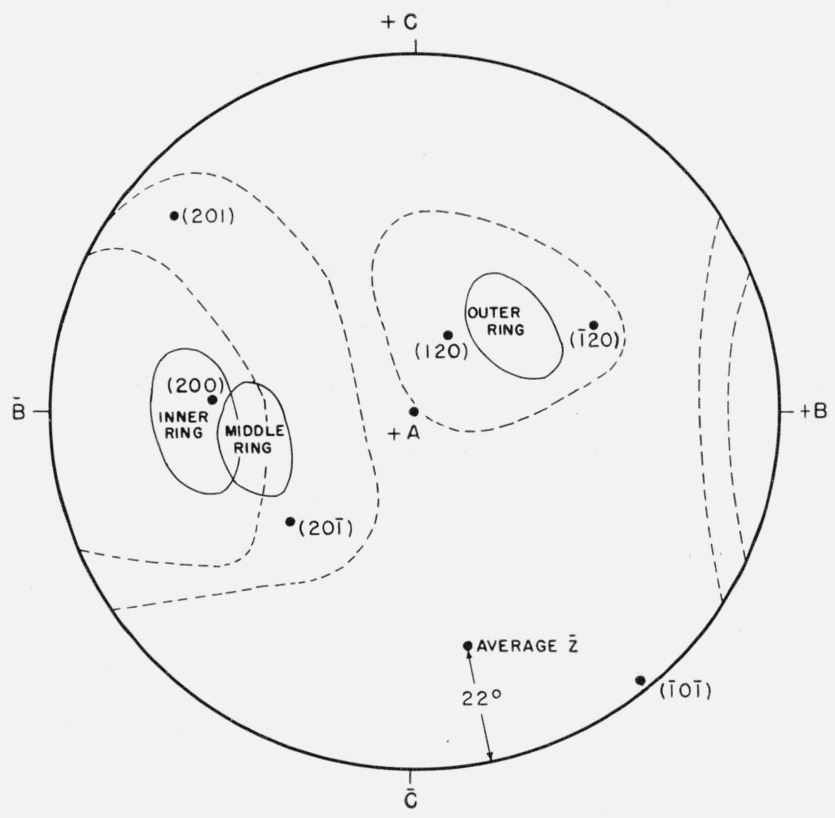

Figure 3. Combined pole figures for stark rubber specimen. inherent in the low-contrast diffraction readings.

It may be of interest to observe that the normal to the (101) crystallographic plane showed a preference to lie parallel to the plane of the sheet of rubber. The authors have not determined whether or not this plane or its normal has any special structural significance in the unit cell. It is interesting to point out, however, that A. Brown [9] found with polyethylene at low extensions (less than 200\%) that the (011) plane normal was preferred in the stretch direction; and W. A. Sisson [5] working with cellulose found, in addition to the major orientating tendency with respect to the cellulose-chain axis, a minor or selective tendency with reference to the (101) plane. It may be that the apparent alinement of the pseudo-dodecahedral plane normal in the stark-rubber sheet is an analogous phenomenon. Strictly speaking, the above interpretation applies only to the small X-ray specimen. There is of course the possibility that, due to the heterogeneity of the original sample, the interpretation with respect to the geometry of the sheet may not be typical of the sample as a whole.

\section{Summary}

It has been shown that the crystallites in stark rubber are not randomly oriented; and, by means of pole figures, the preferred orientation of the particular specimen under study has been herewith presented. A study of the figure reveals that a (101) plane normal lies in the original plane of the rubber sheet, a condition that may be related to observations made with other polymers. The pole-figure method of analysis, of long standing in metallurgical studies, would appear to deserve wider employment in orientation problems of polymers, especially in those cases in which the diffraction patterns do not approach those of fibers.

\section{References}

[1] D. E. Roberts and L. Mandelkern, The nature of stark rubber, J. Research NBS 54, 167 (1955) RP2578.

[2] C. W. Bunn (R. Hill, Ed.) Fibers from synthetic polymers, p. 263 (Elsevier Publishing Co., Amsterdam, 1953).

[3] P. H. Herman, The physics and chemistry of cellulose fibers, p. 296 (Elsevier Publishing Co., Amsterdam, 1949).

[4] W. A. Sisson (E. Ott, Ed.) Cellulose and cellulose deviations, p. 231 (Interscience Publishers, Inc., New York, N.Y., 1946).

[5] W. A. Sisson, X-ray studies of crystallite orientation in cellulose fibers II, J. Phys. Chem. 40, 343 (1936).

[6] C. S. Barrett, Structure of metals, 2d ed., p. 28 (McGrawHill Book Company, Inc., New York, N. Y., 1952).

[7] C. W. Bunn, Molecular structure and rubber-like elasticity, Proc. Roy. Soc. (London) [A] 180, 40 (1942).

[8] B. F. Decker, Preferred orientations in rolled metals: Construction of pole figures, Am. Soc. Testing Materials, Proc. 43, 785 (1943).

[9] A. Brown, X-ray diffraction studies of the stretching and relaxation of polyethylene, J. Appl. Phys. 20, 552 (1949).

Washington, March 24, 1955. 